\title{
Performanance Analysis of Mac Protocols for WBAN on Varying Transmitted Output Power of Nodes
}

\author{
Vinay Singh \\ Assistant Professor \\ Gwalior Engineering College,Gwalior
}

\author{
Rahul Sharma \\ Assistant Professor \\ Gwalior Engineering College, Gwalior
}

\begin{abstract}
The most common protocols for Wireless Body Area Network (WBAN) like TMAC, SMAC, ZigBee MAC, Baseline MAC protocols are analyzed in this work to evaluate the effect of various transmitted output power of nodes. When WBAN is used in patient monitoring, the analysis is required up to a great extent. In critical cases very high traffic load and accuracy of received packets is required while in normal patient health monitoring the traffic load is lower than that of critical case. This analysis is important in point of view of varying requirement of WBAN according to varying conditions. On varying the output power of nodes a considerable effect is observed on throughput and energy consumption for MAC protocols that is presented in the paper.
\end{abstract}

\section{INTRODUCTION}

Wireless Body Area Network (WBAN) is an emerging technology that is serving medical field with comfort and highly reduced cost. In WBAN, one node is network coordinator and all other nodes are arranged in star topology. All these sensor nodes are implanted on patient's body and collecting data related to the patient's health to send it to network coordinator. Network coordinator establishes connections with various medical servers to get proper prescription and to send the information related to patient's health. In this paper only the communication between network coordinator and server nodes is considered. MAC protocols for WBAN are analyzed in this paper to observe the effect of changing the output power of nodes. Some popular MAC protocols are TMAC, ZigBee MAC, SMAC and Baseline MAC. TMAC and SMAC are dutycycling protocols Where SMAC is the previous version of TMAC. ZigBee MAC protocol can use CSMA/CA or TDMA schemes and gives better energy efficiency and throughput in simple WBAN having small number of nodes. Baseline MAC protocol employs CSMA/CA technique and its performance is average in WBAN. These MAC protocols show large variations in their performance when the transmitted output power of nodes is changed. An analysis of these effects is done in this paper.

\section{RELATED WORK}

Several researchers are being attracted towards analysis of performances of MAC protocols in WBAN. In a study of performance of MAC protocols in WBAN several protocols are tested for their compatibility with WBAN. This study suggests that T-MAC, S-MAC, ZigBee MAC, and Baseline MAC protocols perform efficiently in case of WBAN [1].In another research work author analyzed MAC protocols to suggest the most energy efficient protocol for WBAN [3] [5] [6] [8]. In some research works T-MAC protocol is analyzed for its better performance than other protocols [7].

\section{RESEARCH METHODOLOGY}

For analysis of 10 nodes architecture of WBAN is taken which are numbered from 0 to 9 . These nodes are arranged in star topology and all the nodes from 1 to 9 are connected to node 0 which is working as network coordinator. Node 0 is taken as sink in WBAN architecture for isolating it for proper analysis. Throughput and energy consumption are most important parameters for evaluating the performance of WBAN. Throughput is can be interpreted by packets received per node at network coordinator. Latency in receiving packets is also an essential parameter in performance of a MAC protocol. These parameters are analyzed at various output powers for various MAC protocols and results are compared to present the effect of change in output power of nodes.

\section{MAC PROTOCOLS FOR WBAN}

MAC protocols used in WBAN must be low power consuming, accurate and with less latency. The most important thing is the protocol should give good performance on varying traffic load. Some popular protocols for WBAN are TMAC, SMAC, ZigBee MAC and Baseline MAC.

TMAC: It is a duty-cycling protocol. In this protocol the node is awaken for a particular period that is called active time. Duty cycle changes according to the information traffic load of the network. When traffic load is high than the duty cycle becomes large so that nodes can handle high traffic load. When traffic load is low then duty cycle is adjusted to small value so that nodes can save their power reducing the problem of idle listening. TMAC protocol is able to handle varying load with low power consumption.

SMAC: SMAC protocol is similar to TMAC but only difference is its fixed duty cycle. This protocol is the previous version. This protocol is not efficient in handling continuously varying data rates in WBAN.

ZigBee MAC: ZigBee MAC protocol can use two schemesCSMA/CA or TDMA. While using CSMA/CA mechanism this protocol gives average performance but using TDMA mechanism (applying Guaranteed Time Slot or GTS) it reduces the power consumption up to a great extent. At high rates the data loss becomes high in TDMA mechanism so it is best when there is less no of nodes or low traffic load.

Baseline MAC: This MAC protocol uses CSMA/CA scheme. The performance of Baseline MAC in terms of energy consumption is not average but throughput is average.

\section{PERFORMANCE RESULTS AND DATA ANALYSIS}

The performance of various MAC protocols is evaluated by considering the following performance parameters: 


\section{Throughput \\ 2. Latency \\ 3. Consumed Energy}

These are the most important performance parameters while analyzing WBAN as we require ultra low energy consumption with maximum throughput and minimum delay to increase the accuracy and lifetime of WBAN and reduce the delay of critical information. These parameters have a trade-off between each other so it is complicated to meet all requirements at the same time. The effect of varying the transmitted output power is observed further through simulation results.

Effect of Varying Transmission Output Power on Throughput: Throughput of WBAN depends on several factors but factors that are affected by change in Output Power are Interference and Break down of Packets due to low sensitivity of nodes. Throughput is proportional to the number of successfully received packets at network coordinator per node. Here throughput is represented through the Received packets per node at $25 \mathrm{kbps}$ data rate of nodes in Figure 1.

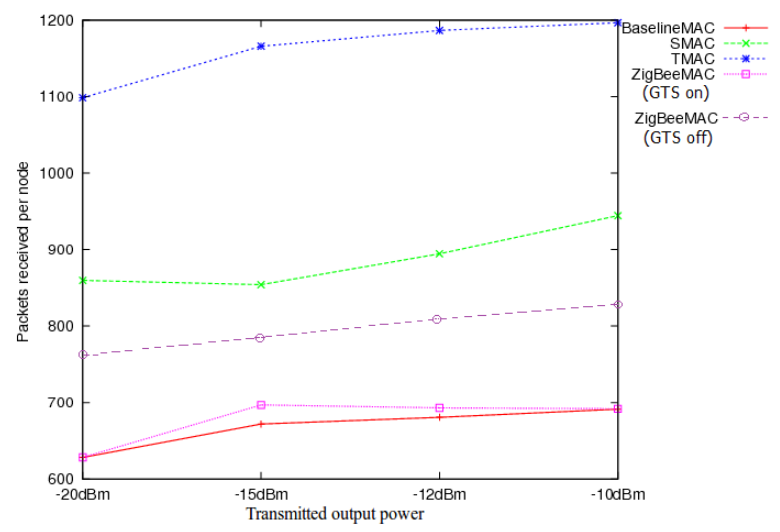

Figure1. Packets received per node

Baseline MAC protocol uses contention based approach so interference plays an important role. Management and control packets are overhead that facilitate efficiency of transmissions at the cost of extra energy consumption. Packet loss due to busy channel, buffer overflow and interference reduces the throughput at a great extent. On increasing the transmitted output power of nodes packet loss due to busy channel and buffer overflow reduces so the throughput improves which is shown in figure. In ZigBee MAC protocol while using contention based CSMA/CA approach, increase in transmitted output power reduces the packet loss due to interference and below sensitivity. Thus throughput of ZigBee MAC when using contention based scheme (or GTS off) increases with transmitted output power of nodes. In ZigBee MAC protocol while using GTS (Guaranteed time slot) or TDMA approach throughput increases with increase in transmitted output power as packet loss due to low sensitivity and buffer overflow reduces. Duty-cycling protocol SMAC performs better than Baseline MAC and ZigBee MAC protocols in terms of throughput. On increasing transmitted output power throughput also increases gradually at $25 \mathrm{Kbps}$ data rate. In SMAC at higher data rates on increase in transmitted output power, the successful transmissions increases and traffic load on channel also increases which causes more collisions of packets. Hence at higher rates like 50Kbps and more, throughput decreases on increasing transmitted output power more than $-15 \mathrm{dBm}$.

Effect of Varying Transmitted Output Power on Latency in Transmission: In general if Latency is considered, Packet loss during transmission is important factor that determine the Latency of WBAN. When transmitted output power varies, a significant variation is observed in packet loss during transmission and hence latency is also affected. In case of TMAC protocol, when transmitted output power is increased from $-20 \mathrm{dBm}$ to $-15 \mathrm{dBm}$, latency increases from $518 \mathrm{~ms}$ (latency is in milli second) to $538 \mathrm{~ms}$ as throughput increases and more number of packets are to be transmitted on the shared medium. But on increasing the output power further from $15 \mathrm{dBm}$, latency reduces negligibly. In SMAC protocol latency is more than that of other protocols due to its fixed duty cycle. With increase in transmitted output power of nodes from $20 \mathrm{dBm}$ to $-15 \mathrm{dBm}$ then latency reduces from $7737 \mathrm{~ms}$ to $7686 \mathrm{~ms}$. On increasing the power further latency increases hence $-15 \mathrm{dBm}$ transmitted output power is the best for WBAN while using SMAC. On the other hand CSMA/CD based ZigBee MAC protocol is the most efficient in terms of latency as it is having latency around $18 \mathrm{~ms}$ on $-15 \mathrm{dBm}$ and on increasing the transmitted output power up to $-12 \mathrm{dBm}$ latency increases as number of packets on medium to transmit are increasing due to less packet breakdown and on increasing further from $-12 \mathrm{dBm}$ to $-10 \mathrm{dBm}$, latency reduces as number of successful transmissions of packets increase. In ZigBee MAC while using GTS (TDMA approach) latency increases on increase in output power up to $-12 \mathrm{dBm}$ and on further increasing output power latency reduces same as that of in ZigBee MAC using CSMA/CD but only difference is that latency in TDMA approach is more than that of ZigBee MAC using CSMA/CD as node has to wait for its assigned time slot. In Baseline MAC protocol latency is reduced on increasing transmitted output power because successful transmissions are increased with increase in transmitted output power.

Effect of Varying Transmitted Output Power on Energy consumption: It is Obvious that on increasing the transmitted output power of nodes, energy consumption of the WBAN will not effected up to a great extent. This fact is also clear in the graph where

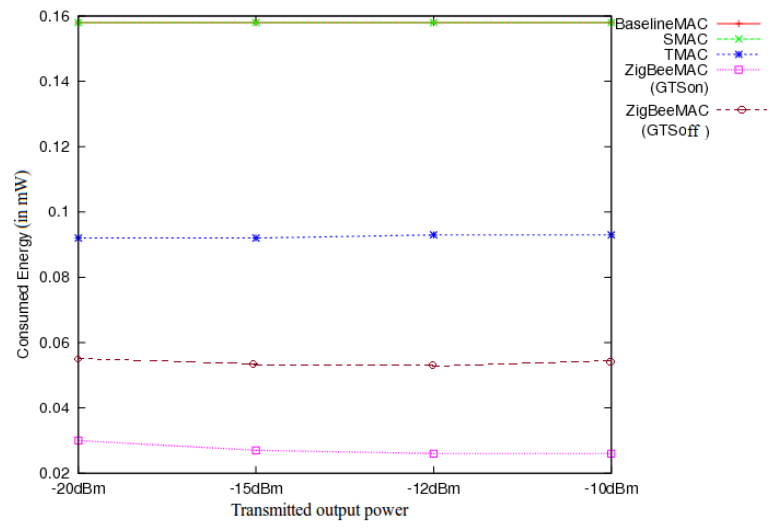

it is clear that overall no significant change in energy consumption is observed because when transmitted power is increased successful transmissions increases and the increase in energy consumption is balanced by the energy saved by protocols by reducing the packet loss. On comparing the energy consumed by various protocols it is found that SMAC and 
Baseline MAC consuming more energy than TMAC and ZigBee MAC. SMAC uses fixed duty cycle and contention based scheme for transmission of packets. So packet loss causes extra energy consumption to retransmit the lost packets. In Baseline MAC protocol the overhead of management and control packets and contention based approach reduce its energy efficiency even less than that of SMAC.

\section{CONCLUSION}

In this paper it is obvious after analyzing the results that the best transmitted output power of nodes must be $-15 \mathrm{dBm}$. At this power the performance of the WBAN with all four protocols is good in every term. At $-20 \mathrm{dBm}$ packet loss occurs as power of many packets is below sensitivity of node. At higher output powers like $-12 \mathrm{dBm}$ and $-10 \mathrm{dBm}$ packet loss due to interference dominates and performance is degraded.

\section{REFERENCES}

[1] Ullah S., Shen B., Riazul Islam S., Khan P., Saleem S., Sup Kwak K., "A Study of MAC Protocols for WBANs", published in Sensors,2010; vol. 10(1), pp. 128-145.

[2] Li, C.; Li, H.-B.; Kohno, R. "Performance evaluation of IEEE 802.15.4 for wireless body area network." In Proceedings of IEEE International Conference on ICC Workshops, Dresden, Germany, 2009, pp.1-5.

[3] Fang, G.; Dutkiewicz, E. BodyMAC: Energy efficient TDMA-based MAC protocol for wireless body area networks. In Proceedings of IEEE ISCIT 2009, Incheon, Korea, 2009.

[4] Li, H.M.; Tan, J.D. "Heartbeat driven MAC for body sensor networks". In Proceedings of ACM SIGMOBILE International Workshop on Systems and Networking Support for Healthcare and Assisted Living Environments, San Juan, Puerto Rico, 2007. pp. 25-30.

[5] Kohvakka, M.; Suhonen, J.; Hamalainen, T.D.; Hannikainen, M. Energy-efficient reservationbasedmedium access control protocol for wireless sensor networks. EURASIP J. Wireless Communication Networks 2010, 22.

[6] Ye. W.; Estrin, H.J. An energy-efficient MAC protocol for wireless sensor networks. In Proceedings of Twenty-First Annual Joint Conference of the IEEE Computer and Communications Societies, Miami, FL, USA, 2002; pp. $1567-1576$

[7] Niamat Ullah *, Pervez Khan and Kyung Sup Kwak "A Very Low Power MAC (VLPM) Protocol for Wireless Body Area Networks", ISSN 1424-8220, Sensors 2011, 11, pp. 3717-3737; doi:10.3390/s110403717.

[8] Timmons, N.F.; Scanlon, W.G. Analysis of the performance of IEEE 802.15.4 for medical sensor body area networking. In Proceedings of First Annual IEEE Communications Society Conference on Sensor and Ad Hoc Communications and Networks, Santa Clara, CA, USA, October 2004; pp. 4-7.

[9] Van Dam, T.; Langendoen, K. An adaptive energy efficient MAC protocol for WSNs. In Proceedings of ACM Conf. on Embedded Networked Sensor Systems, SenSys 2003, Los Angeles, CA, USA, 2003; pp. 171-180.

[10] Zhen, B.; Li, H.B.; Kohno, R. IEEE body area networks for medical applications. In Proceedings of IEEE International Symposium on Wireless Communication
Systems 2007, Trondheim, Norway, 16-19 October 2007.

[11] Ye. W.; Estrin, H.J. An energy-efficient MAC protocol for wireless sensor networks. In Proceedings of Twenty-First Annual Joint Conference of the IEEE Computer and Communications Societies, Miami, FL, USA, 2002; pp. 1567-1576.

[12] Khan, N.P.; Boncelet, C. PMAC: Energy efficient medium access control protocol for wireless sensor networks. In Proceedings of IEEE Military Communications Conference, Washington, DC, USA, 2006; pp. 1-5, 23-25.

[13] Rajendran, V.; Garcia-Luna-Aveces, J.J.; Obraczka, K. Energy-efficient, application-aware medium access for sensor networks. In Proceedings of IEEE Mobile Adhoc and Sensor Systems Conference, Washington, DC, USA, 2005; p. 8630.

[14] Heinzelman, W.R.; Chandrakasan, A.; Balakrishnan, H. Energy-efficient communication protocol for wireless microsensor networks. In Proceedings of the 33rd Annual Hawaii International Conference on System Sciences, Maui Kula, HI, USA, 2000.

[15] D. Stevanovic and N. Vlajic, "Performance of IEEE 802.15.4 in wireless sensor networks with a mobile sink implementing various mobility strategies," in Local Computer Networks, 2008. 33rd IEEE Conference on, Oct. 2008, pp. 680-688.

[16] L. Huaming and T. Jindong, "Heartbeat driven medium access control for body sensor networks," in Proceedings of HealthNet '07. ACM, 2007, pp. 25-30.

[17] B. A. Kadrovach and G. B. Lamont. A particle swarm model for swarm-based networked sensor systems. In Proc. ACM Symposium on Applied Computing, pages 918-924. ACM, 2002.

A. Milenkovic, C. Otto, and E. Jovanov, "Wireless sensor networks personal health monitoring: Issues and an implementation," Computer Communications, Wireless Sensor Networks and Wired/Wireless Internet Communications, vol. 29, no. 13-14, pp. 2521-2533, August 2006.

[18] H. Cao, V. Leung, C. Chow, and H. Chan, "Enabling technologies wireless body area networks: A survey and outlook," Communications Magazine, IEEE, vol. 47, no. 12, pp. 84-93, Dec. 2009.

[19] S. Pentland, "Healthwear: Medical technology becomes wearableComputer, vol. 37, pp. 42-49, 2004.

[20] World Health Organization [online] http://www.who.int/mediacentre/factsheets/fs317/en/index. htm

[21] International Diabetes Federation (IDF) [online] http://www.idf.org/.

[22] S.Park and S. Jayaraman, "Enhancing the quality of life through wearable technology", IEEE Engineering in Medicine and Biology Magazine, vol. 22, no. 3, pp. 41-48; May-June, 2003.

[23] TG6 Body Area Networks (BAN) draft standards, IEEE 802.15-10-0245-06-0006. http://www.ieee802.org/15/pub/TG6.html 